\title{
Editorial
}

\section{Crowdsensing and Vehicle-Based Sensing}

\author{
Carlos T. Calafate, ${ }^{1}$ Celimuge Wu, ${ }^{2}$ Enrico Natalizio, ${ }^{3}$ and Francisco J. Martínez ${ }^{4}$ \\ ${ }^{1}$ Department of Computer Engineering (DISCA), Universitat Politècnica de València, Camino de Vera S/N, 46022 Valencia, Spain \\ ${ }^{2}$ Graduate School of Information Systems, The University of Electro-Communications, 1-5-1 Chofugaoka, Chofu, Tokyo 182-8585, Japan \\ ${ }^{3}$ Heudiasyc UMR CNRS 7253, Sorbonne Universités, Université de Technologie de Compiègne, CS 60319, \\ 60203 Compiègne Cedex, France \\ ${ }^{4}$ Computer Science and System Engineering Department, University of Zaragoza, Escuela Universitaria Politécnica de Teruel, \\ Ciudad Escolar s/n, 44003 Teruel, Spain
}

Correspondence should be addressed to Carlos T. Calafate; calafate@disca.upv.es

Received 24 October 2016; Accepted 25 October 2016

Copyright (C) 2016 Carlos T. Calafate et al. This is an open access article distributed under the Creative Commons Attribution License, which permits unrestricted use, distribution, and reproduction in any medium, provided the original work is properly cited.

Improvements in terms of smart device capabilities and of communication technologies allowed crowdsensing solutions to emerge as a powerful strategy to revolutionize environment sensing, becoming one of the key elements of future smart cities. In particular, smartphones, smartwatches, and other personal gadgets are now endowed not only with significant computing power and different wireless interfaces, but also with an increasing number of sensors able to provide useful information about the user environment, especially when the user is moving around a city. If vehicular mobility is adopted, the sensing capabilities can be further increased by connecting smart devices to vehicles using the On Board Diagnostic Interface (OBD-II) or provided directly by smart vehicles through vehicle-to-vehicle (V2V) or vehicle-toinfrastructure (V2I) communications. In the future, with the gradual introduction of autonomous vehicles and unmanned aerial vehicles, many more sensing alternatives are expected.

In parallel to these developments, network infrastructure has experienced significant improvements in recent years in terms of both coverage and performance which, combined with advances in cloud computing, allows transmitting, storing, and processing large amounts of data in an efficient manner. Altogether, the potential for generating and analyzing huge amounts of data is remarkable, being able to provide unprecedented information with high levels of spatial and time resolution about any event of interest occurring in a city.

The purpose of this special issue is to address recent advances on mobile sensing, with particular emphasis on novel applications and architectures for crowdsensing and vehicle-based sensing.

The articles contained in the present issue include both reviews and research studies focused on data sensing and processing in situations where either pedestrians or vehicles act as mobile sensors.

As an introduction to the topic, the paper by W. Zamora et al. entitled "A Survey on Smartphone-based Crowdsensing Solutions" provides a survey of smartphone-based crowdsensing solutions that have emerged in the past few years, focusing on different works published in top-ranked journals and conferences. To properly analyze these previous works, they define a reference framework that allows classifying the different proposals under study. Globally, the survey provides useful insight into the broad scope of the crowdsensing area, being of interest to both experts and novices.

Analyzing and optimizing mobility in the urban domain is one of the most relevant contributions that mobile crowdsensing can offer to smart cities. In particular, the everincreasing capabilities of smartphones and vehicle-mounted devices pave the way for gaining meaningful insight into urban mobility. In this context, the work by F. Terroso-Sáenz et al. entitled "Human Mobility Modelling Based on Dense Transit Areas Detection with Opportunistic Sensing" introduces a novel approach to leverage such paradigm, allowing composing a map of Dense Transit Areas (DTAs) within a city representing some of its mobility features while protecting the privacy of users. Besides vehicles, pedestrian and 
multimodal paths would also benefit greatly from context data, as well as the information about the whole experience of traveling and wandering the city, including travel planning and payments. The work by S. Mirri et al. entitled "A Service-Oriented Approach to Crowdsensing for Accessible Smart Mobility Scenarios" introduces a prototype of the infrastructure and overall architecture proposed to meet these challenges, describing some of the services that can be provided: path recommendations for wheelchair users and for elderly persons.

In addition to mobility optimization, traffic safety is also a critical issue that should be addressed to minimize the chances of accidents. In this context, the work by Z. Liu et al. entitled "SenSafe: A Smartphone-Based Traffic Safety Framework by Sensing Vehicle and Pedestrian Behaviors" proposes a driving behavior detection mechanism that relies on smartphones to sense events in the proximity and provide alerts to drivers. Their proposal also includes a low-overhead approach for fast data broadcasting, along with a collision estimation algorithm to trigger warnings when dangerous situations are detected. Since broadcasting is a key element for security and context awareness in vehicular ad hoc environments by simplifying direct vehicle-to-vehicle communications, effective information exchanging becomes critical. The work by C. Chen et al. entitled "A Safety Enhancement Broadcasting Scheme Based on Context Sensing in VANETs" addresses such problem by proposing a congestion control scheme for vehicular networks that can dynamically adapt to variable channel occupation to maximize the network throughput while avoiding to congest the channel, thereby improving network throughput, packet delivery ratio, and transmission delay.

Finally, the efficient handling of data itself gains utmost importance in the crowdsensing context, including both data acquisition and dissemination. Concerning the latter, novel paradigms have recently emerged, as is the case of the floating data paradigm which aims at enabling a floating data network in a distributed and collision-free way. The work by A. Bujari and C. E. Palazzi entitled "AirCache: A Crowd-Based Solution for Geoanchored Floating Data" is an example of such a solution, whose aim is to guarantee data availability in an Area of Interest while reducing the data access costs at the network edges. In particular, it relies on replication among passing-by or stationary users, in addition to a node election strategy, to improve the global energy/memory consumption needed to maintain the data floating.

With respect to the data gathering process, especially when using smartphones, it usually involves different data types due to the different granularity, multiple sensor sources, and time labelling. Such heterogeneity and time dependencies introduce new challenges in the data analysis process. The work of C. Ma et al. entitled "Representation Learning from Time Labelled Heterogeneous Data for Mobile Crowdsensing" addresses this challenge by proposing a new representation learning method for heterogeneous data with time labels that allows extracting typical features using deep learning. In their work they exemplify the applicability of their proposed method by differentiating between two mobile activities using smartphone sensors: walking versus cycling and driving versus taking the bus.

Overall, we hope that this special issue is able to shed some light on the major developments in the area of crowdsensing and vehicle-based sensing and attract the attention of the scientific community to undertake further investigations leading to the rapid deployment of such novel solutions.

\section{Acknowledgments}

We would like to express our appreciation to all the authors for their novel contributions and the reviewers for their support and constructive critiques in making this special issue possible.

Carlos T. Calafate Celimuge $\mathrm{Wu}$

Enrico Natalizio

Francisco J. Martínez 

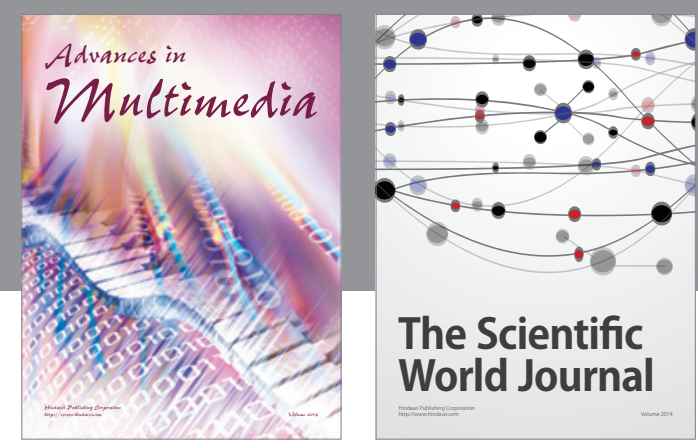

The Scientific World Journal
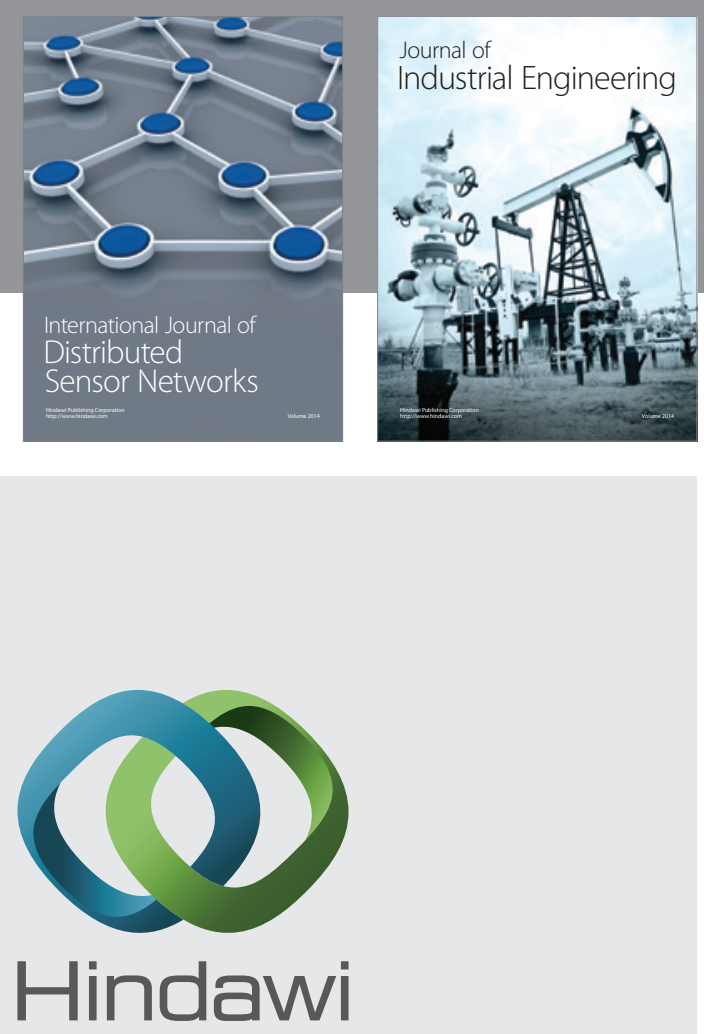

Submit your manuscripts at

http://www.hindawi.com

\section{Computer Networks} and Communications
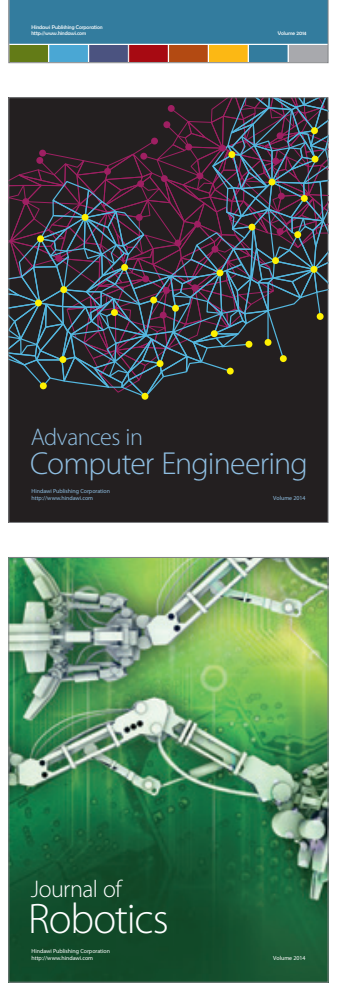
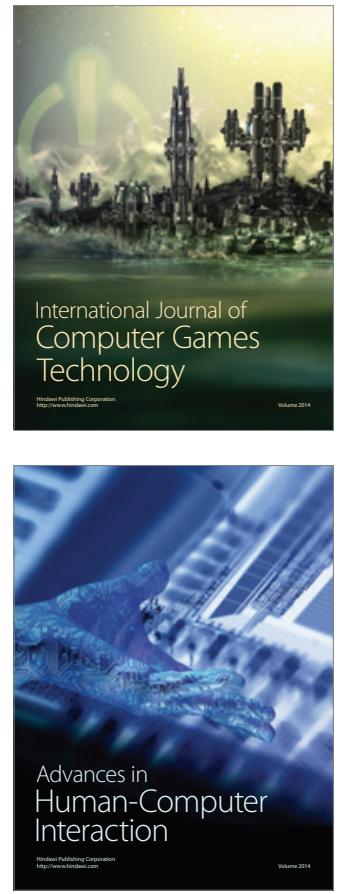
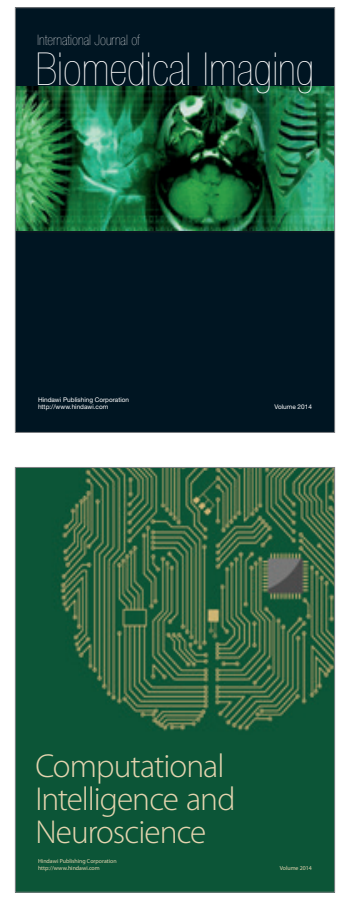
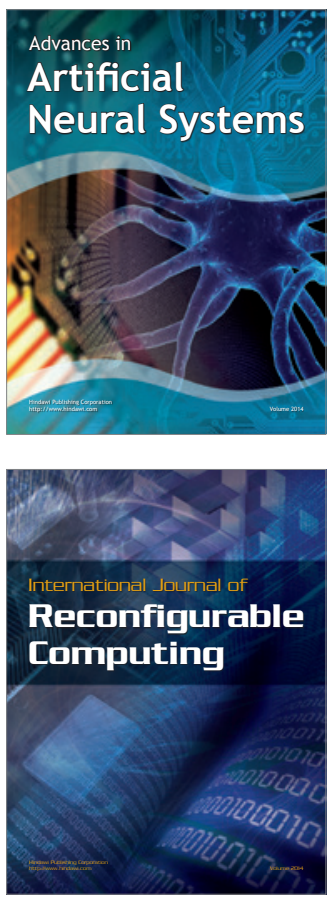
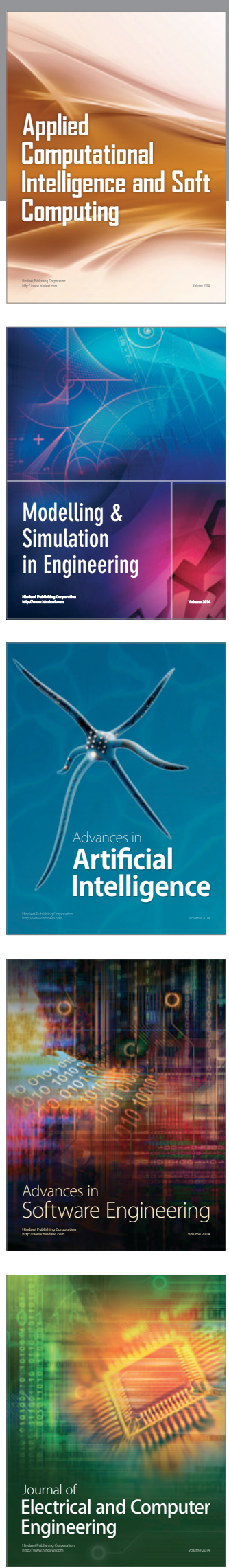